\title{
Simple Balanced Binary Search Trees
}

\author{
Prabhakar Ragde \\ Cheriton School of Computer Science \\ University of Waterloo \\ Waterloo, Ontario, Canada \\ plragde@uwaterloo.ca
}

\begin{abstract}
Efficient implementations of sets and maps (dictionaries) are important in computer science, and balanced binary search trees are the basis of the best practical implementations. Pedagogically, however, they are often quite complicated, especially with respect to deletion. I present complete code (with justification and analysis not previously available in the literature) for a purely-functional implementation based on AA trees, which is the simplest treatment of the subject of which I am aware.
\end{abstract}

\section{Introduction}

Trees are a fundamental data structure, introduced early in most computer science curricula. They are easily motivated by the need to store data that is naturally tree-structured (family trees, structured documents, arithmetic expressions, and programs). We also expose students to the idea that we can impose tree structure on data that is not naturally so, in order to implement efficient manipulation algorithms. The typical first example is the binary search tree. However, they are problematic.

Naive insertion and deletion are easy to present in a first course using a functional language (usually the topic is delayed to a second course if an imperative language is used), but in the worst case, this implementation degenerates to a list, with linear running time for all operations. The solution is to balance the tree during operations, so that a tree with $n$ nodes has height $O(\log n)$. There are many different ways of doing this, but most are too complicated to present this early in the curriculum, so they are usually deferred to a later course on algorithms and data structures, leaving a frustrating gap. Furthermore, most presentations in conventional textbooks avoid recursion in favour of loops, use mutation to implement local rotations, and often, due to space constraints, present final optimized code rather than intermediate versions that might aid in understanding.

A purely functional approach to data structures usually facilitates educational discussion, and it comes close in this case, but does not close the gap. The invariants for red-black trees [5] (an encoding of 2-3-4 trees, which can be viewed as a special case of B-trees) are difficult to motivate, but the code for maintaining it in the case of insertion is short and easy to justify, as Okasaki showed [10].

Okasaki's code for insertion into a red-black tree applies a balance function to each tree constructed with the result of a recursive call. His compelling contribution was to notice that the heart of the rebalancing function in the case of insertion consists of five cases, each one line long and exhibiting a pleasing symmetry (the right-hand sides of each case can be made identical). However, he does not handle deletion, with good reason; it is much more complicated.

Stefan Kahrs added code for deletion from red-black trees [8] that is the basis for a Haskell [6] library available through Hackage [4], but his code is complex, and comes with no explanation. An explanation of related code is available in a journal paper [7], but his goal in this paper was to use advanced features of the Haskell type system to enforce invariants, rather than to ease matters for undergraduates. Matthew

J. Caldwell, Ph.K.F. Hölzenspies, P. Achten:

Trends in Functional Programming in Education (TFPIE 2014)

EPTCS 170, 2014, pp. 78-87, doi $10.4204 /$ EPTCS.170.6

(c) P. Ragde 
Might, in a lengthy blog post, calls code based on Kahrs's work ported to other languages such as Scala "Byzantine"[9].

Might tackles the topic from a pedagogical point of view, introducing two new colours (double-black and negative black) during the deletion phase, a notion of colour arithmetic, customized match expanders for his Racket [11] implementation, and three code phases (removing, bubbling, rebalancing). This is probably the best presentation of deletion from red-black trees, but it is still not good enough to close the gap; the code is still too long, and the justification too complicated.

The simplest deletion code known (addition is also simple) is for AA trees, an encoding of 2-3 trees, named after their creator Arne Andersson. Andersson's work [2] was published in a conference with a restriction on page length, and while it is available on his Web site, there is no journal version or longer exposition. The paper gives the invariants but does not explain how the code maintains them. The code is written in Pascal; it does use recursion (he apologizes for this to his audience) but it also makes heavy use of mutation.

Here we will work out a purely functional implementation. To the best of my knowledge, this is the first publication of a purely-functional treatment of AA trees with complete justification and analysis, and it is simpler than any of the work cited above.

\section{Invariants}

As mentioned in the previous section, AA trees are an encoding of 2-3 trees [1]. A 2-3 tree generalizes a binary tree, a node of which contains one key $k$, a left subtree with keys less than $k$, and a right subtree with keys greater than $k$. (We assume unique keys, but it is easy to adapt the code to handle duplicates.) 2-3 trees allow ternary nodes containing two keys $k_{1}, k_{2}$ and three subtrees: a left subtree with keys less than $k_{1}$, a middle subtree with keys between $k_{1}$ and $k_{2}$, and a right subtree with keys greater than $k_{2}$. In contrast to the naive elementary implementation of binary search trees, 2-3 trees do not allow nodes with a single child, and all leaves are at the same depth.

2-3 trees are a good approach to understanding the concepts behind balanced binary search trees and help to explain the invariants of both red-black trees and AA trees. However, code implementing them directly gets complex due to the number of special cases. Consequently, it makes sense to simulate them by binary search trees. A node in the 2-3 tree is simulated by one or two binary nodes in the AA tree. Instead of a colour at each node (as is the case with the red-black simulation of 2-3-4 trees), we maintain the level that the key would be at in the 2-3 tree. An empty tree has level 0 , leaves have level 1 , their parents have level 2, and so on. Besides simplifying the code, this also aids students in understanding how and why invariants need to be maintained.

An AA tree is a binary search tree, and so the code for searching is unchanged from the naive implementation (as is the case for all balanced binary search tree schemes). To ensure that an AA tree actually does encode a 2-3 tree, it is necessary to maintain some other invariants as well. 
In the following diagrams illustrating the invariants, a node is labelled with its key, and levels decrease from top to bottom.

AA1: The left child of a node $x$ has level one less than $x$.

\section{5}

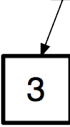

AA2: The right child of $x$ has the same level ( $x$ is a "double" node) or one less ( $x$ is a "single" node).

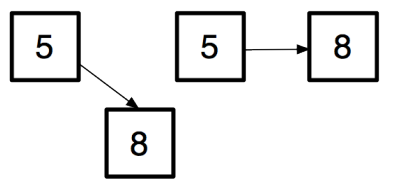

AA3: The right child of the right child of $x$ has level less than $x$.

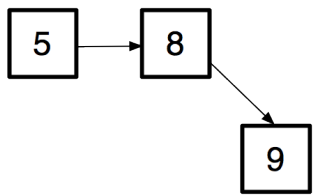

AA4: All internal nodes have two children.

The invariants ensure that the height of the AA tree is logarithmic in the number of nodes. In the 2-3 tree being simulated, all internal nodes have two or three children. Internal 2-3 nodes with three children are simulated by an double AA tree node and its right child.

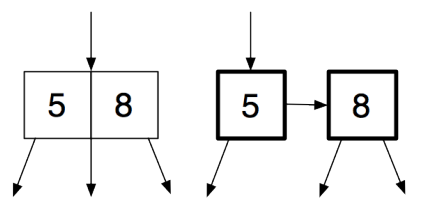

Leaves of the 2-3 tree may contain one or two values, and all leaves are at the same depth from the root. This means that the height of the 2-3 tree is logarithmic in the number of nodes. As with red-black trees, a root-node path in the AA tree is at most twice as long as the corresponding path in the 2-3 tree, thanks to invariant AA3.

\section{Insertion}

For brevity, I will use Haskell in this exposition, though nothing in the code requires laziness or static typing. A Racket implementation (Racket is strict and dynamically typed) is also brief compared to the other options; it is slightly more verbose than the code presented here due to differences in syntax, not semantics. We start developing the code using an elementary implementation of binary search trees without balancing (and thus no levels to maintain), as would be seen in a typical introductory course. 
In the code and in the diagrams, we will follow the convention that we will call a tree argument $t$ where possible, and subtrees will be referred to by letters from the beginning of the alphabet ( $a$, b, etc.), in lower case as is required in Haskell. Individual nodes will be referred to by letters from the end of the alphabet (y, $\mathrm{z}$, etc.). If we need to pattern-match to gain access to individual fields of the node, we will use the convention that node $t$ will have level lvt, left subtree lt, key kt, and right subtree $r t$.

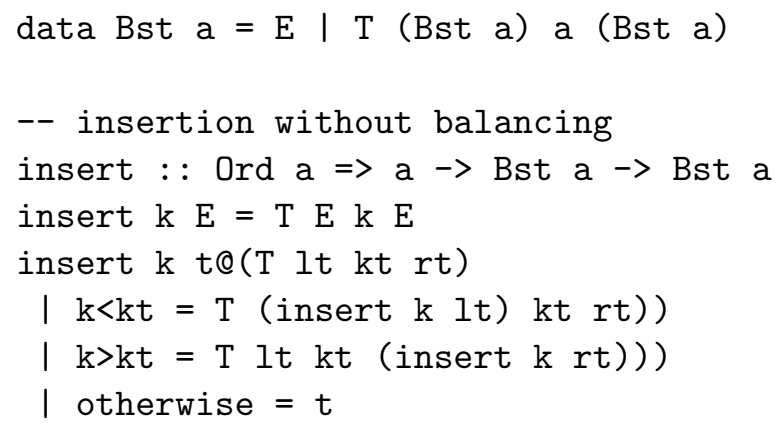

As with Okasaki's code for insertion, we will rebalance each tree constructed with the result of a recursive application. We will split the rebalancing function for AA trees into two two-line helper functions, skew and split, which will also be useful for deletion. (These functions appear in Andersson's paper with the same names, though in imperative versions that use mutation.) Note the introduction of the integer level argument for the $\mathrm{T}$ data constructor.

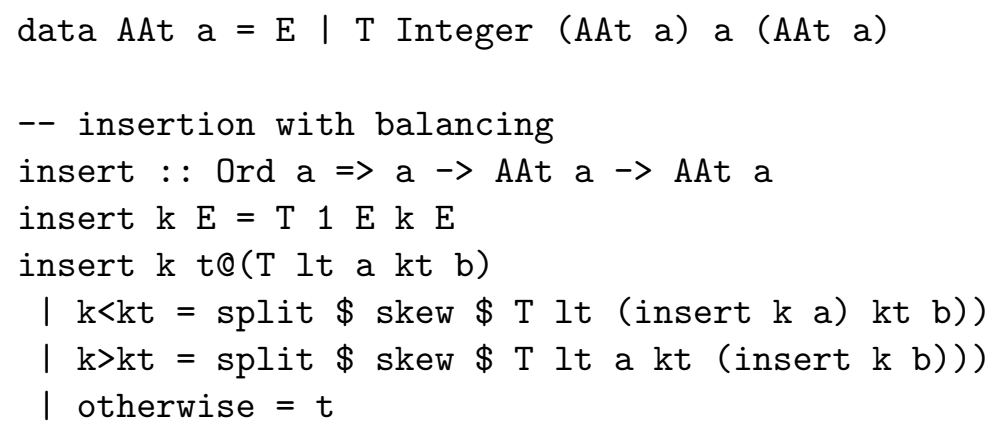

In general, a subtree produced as the result of a recursive application of insert may have had the level of its root increased by one, and means that the tree constructed from it by adding in the unchanged left or right subtree may not satisfy the invariants.

If the left child of a node is increased by one, it is single (as we will see), and we need the skew transformation to fix the violation of invariant AA1. (A diagram showing a pair of trees, such as shown below, represents "before" and "after" situations.)

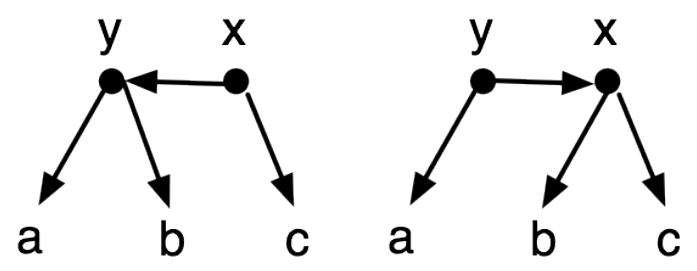

skew ( $\mathrm{T} \operatorname{lvx}(\mathrm{T} \operatorname{lvy} \mathrm{a} \mathrm{ky} \mathrm{b}) \mathrm{kx} \mathrm{c}) \mid(\operatorname{lvx}==\operatorname{lvy})=\mathrm{T} \operatorname{lvx} \mathrm{a} \mathrm{ky}(\mathrm{T} \operatorname{lvx} \mathrm{b} \mathrm{kx} c)$ skew $t=t$ 
But the result of skewing could still be a problem if the former root had a right child at the same level, because invariant AA3 is violated.
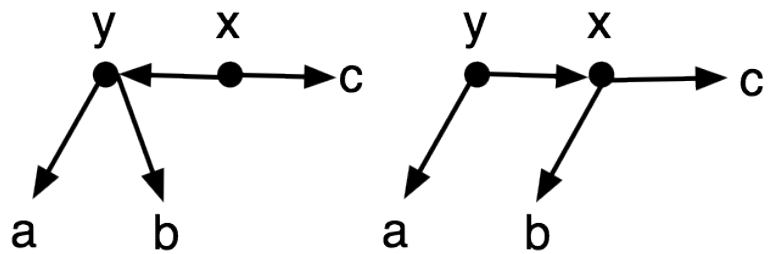

This is also a problem if the right child of a right child at the same level has its level raised. Once again, invariant AA3 is violated after the skew.

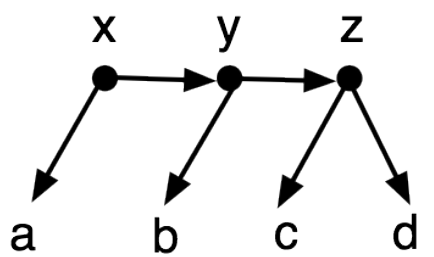

These situations are handled by the split transformation, which solves the problem by raising the level of the middle node by one, ensuring that it is single and the parent of the other two nodes. All invariants are restored.
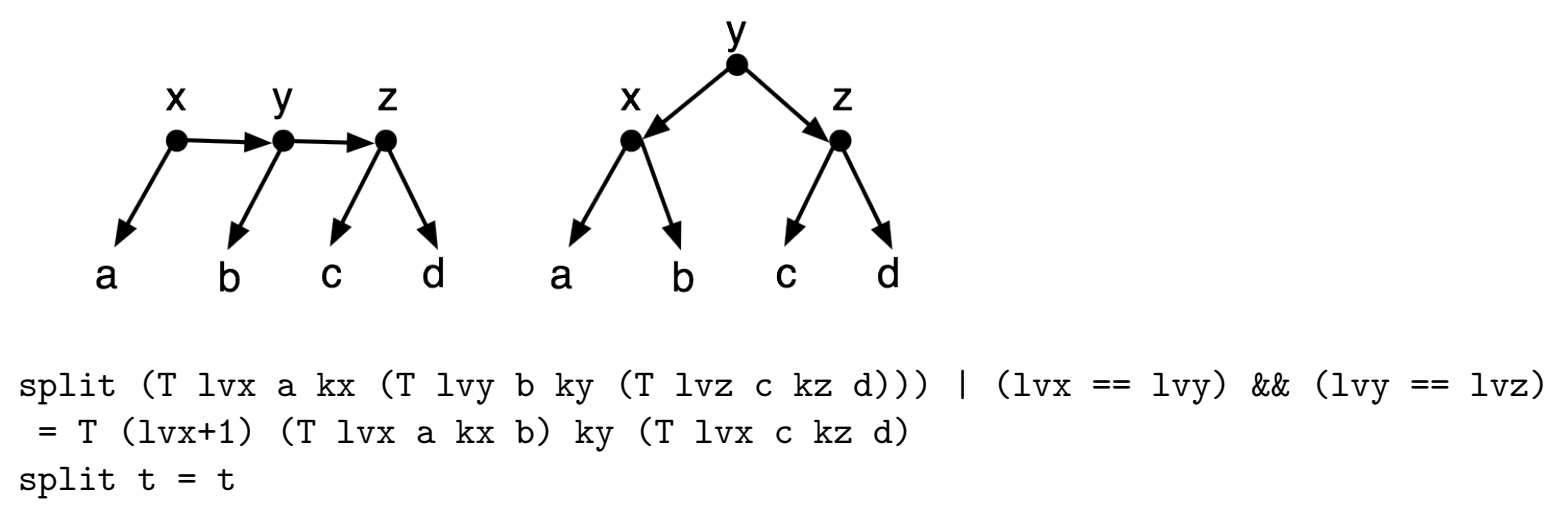

Note that it does not hurt to apply skew and split to trees that do not require those transformations. This completes the presentation of insertion, which so far is at least as short and understandable as Okasaki's, if not more so. The instructor may choose to stop at this point, leaving deletion as a rather challenging exercise.

\section{Deletion}

As with insertion, we start with the code for elementary deletion from an unbalanced binary search tree. The case that is not straightforward occurs if the key to be deleted is in an internal node. In this case, we can, in effect, move the largest key in the left subtree up to replace the key to be deleted. The dellrg helper function deletes the largest key in a binary search tree, and produces a tuple of that key and the new tree. 


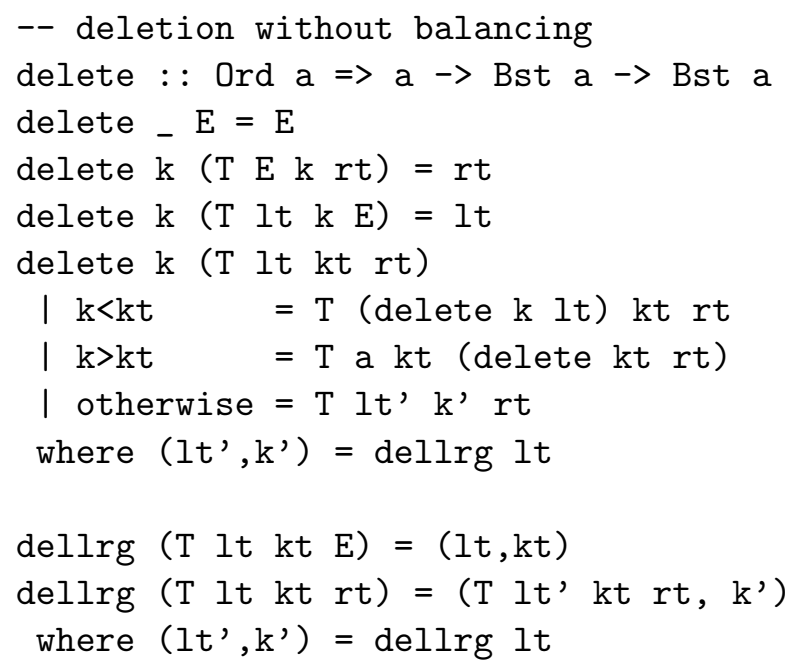

Deletion from an AA tree is more complex than insertion, and we put all the rebalancing logic into the adjust function. This does not have to be applied when one child of the key to be deleted is empty, as the other child is unchanged and must be at level one. But it is applied to trees constructed from the result of recursive applications, including in the dellrg helper function.

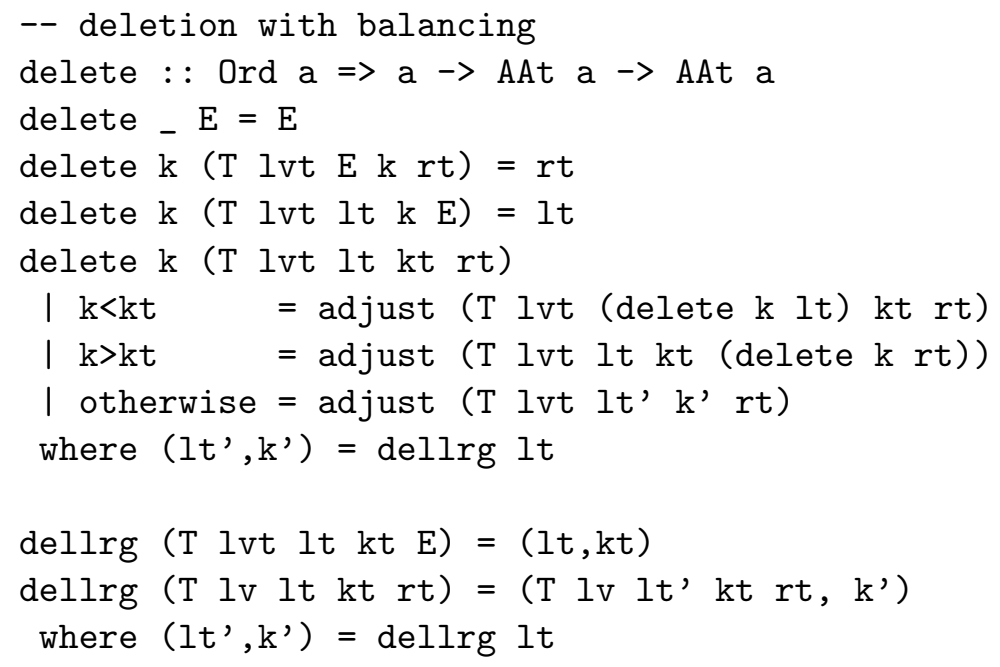

The code for adjust is complicated, and requires careful case analysis to understand. It is still shorter and clearer than deletion for any other balanced binary search tree implementation.

First, some helper functions to make the code a bit shorter and clearer. lvl produces the level of the root of a AA tree, and sngl tests whether the root is single or double.

$\operatorname{lvl} \mathrm{E}=0$

lvl (T lvt _ _ _) $=$ lvt

sngl $E=$ False

sngl $(T+\ldots$ - $E)=$ True

sngl ( $\operatorname{lvx} \ldots$ _ ( I lvy _ _ _ )) = lvx > lvy 
How can the invariant be broken by recursive deletion? A child node may have its level lowered. In general, a node being adjusted may have one child two levels lower (as the result of the child being constructed using the result of a recursive deletion), which violates one of the invariants AA1 or AA2. We might need to drop the level of such a node to restore that invariant.

We are now ready to describe adjust. The easiest case is when each child of the argument tree $t$ has level no lower than one less than the level of $t$. In this case, no adjustment is needed.

adjust $t @(T$ lvt lt $\mathrm{kt} r \mathrm{rt}) \mid(\operatorname{lvl} \mathrm{lt}>=\operatorname{lvt}-1) \& \&(\operatorname{lvl} \mathrm{rt}>=\operatorname{lvt}-1)=\mathrm{t}$

If the right child of $t$ has level two below $t$, there are two subcases, depending on whether the left child of $t$ ( $x$ in the diagram) is single or double. The easier subcase is when $x$ is single. We can drop the level of $t$ by one, fixing the violation of AA2, but this would result in a violation of AA3, since $t$ would then be at the same level as its left child. But a skew operation restores the invariant AA3.
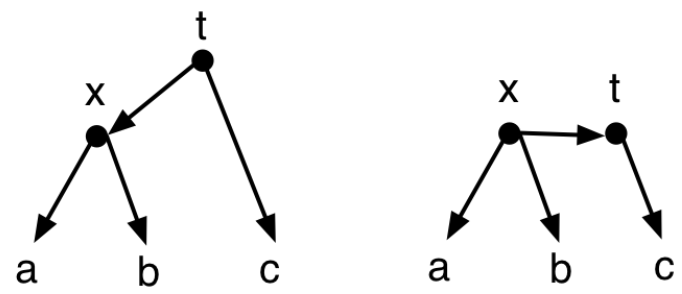

The result is just a skew of $t$ with its level lowered by one.

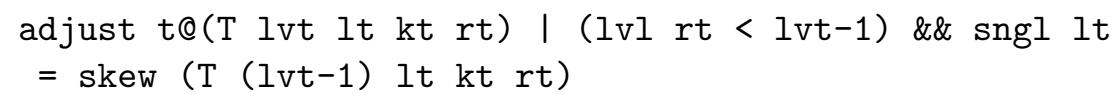

The harder subcase is when the left child of $t$ is double. We need to do a more complicated restructuring.
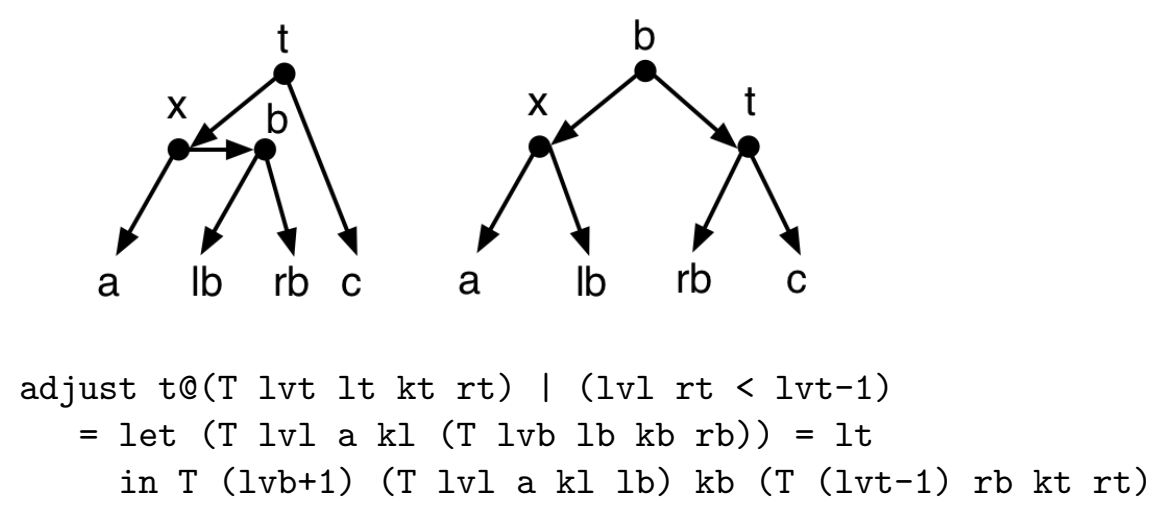

The remaining case of adjust is when the left child of $t$ has level two below $t$. Here again, there are subcases.

The first subcase is when $t$ is single. We can drop its level, but if its right child is double, this will violate invariant AA3. But this is exactly what split was designed to fix.

adjust $t @(T$ lvt lt $k t r t) \mid(l v l ~ r t<l v t)=\operatorname{split}(T(l v t-1)$ lt kt rt) 
The second subcase is when $t$ is double, so $t$ and its right child y are on the same level. We can drop the level of both $t$ and $y$ by one, but then we have a mess, because this might result in as many as five nodes on the same level ( $t, y, a, b$, and $d$ in the diagram below) and multiple violations of invariants. (In the diagram below, $a$ is drawn as double, but it might be single.)

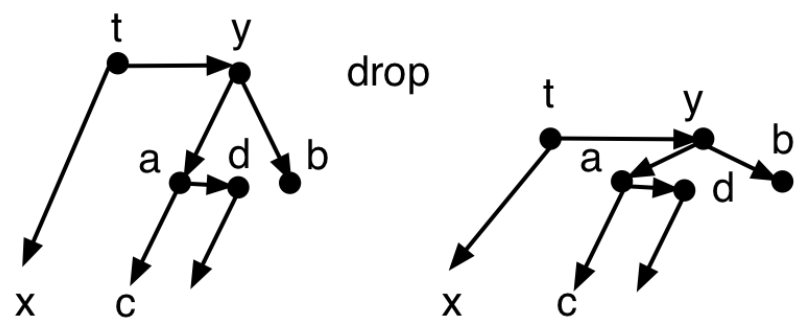

We can explain the fixing up in terms of skew and split, though we don't use them in the final code for this case. We start by naming the various values we need to manipulate, based on the above diagram.

There are two sub-subcases of the second subcase, depending on whether a is single or double. First, we consider the sub-subcase when a is single. In this case, a skew followed by a split fixes things up.
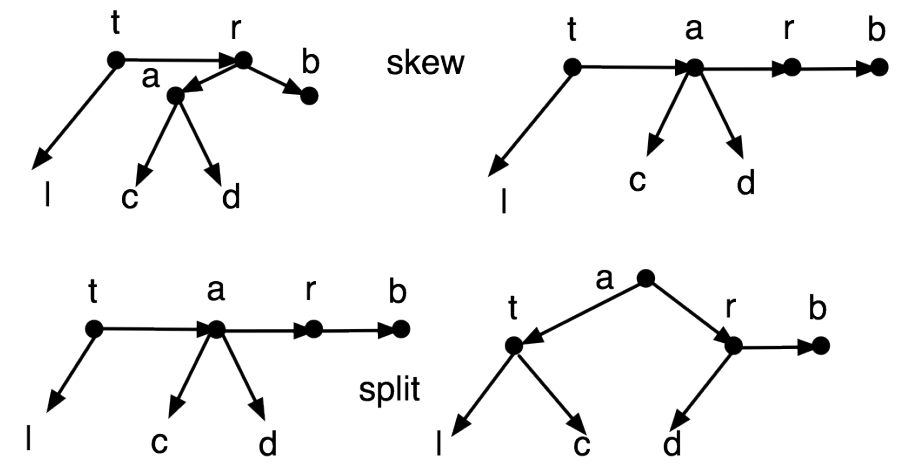

If $\mathrm{b}$ was originally double, it might need to be split at this point.

The sub-subcase where a is double is similar, but two skews and two splits are needed, and $r$ ends up at a different level.
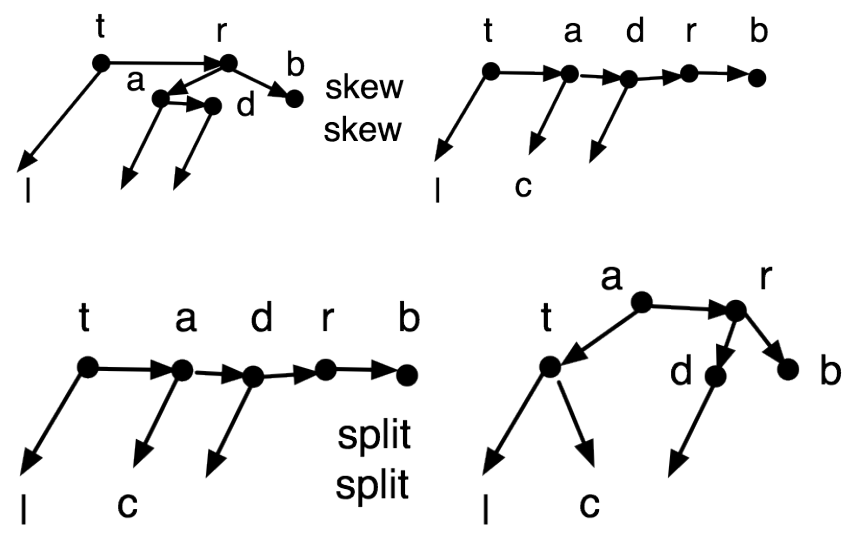

The code for deletion in Andersson's paper applies mutating skew three times and mutating split twice (sometimes to different nodes, so it cannot be written as function composition). His implementation collapses some cases to yield shorter code, at the cost of understanding. Rather than do that here, let's take a look at the end results of the single and double sub-subcases. 


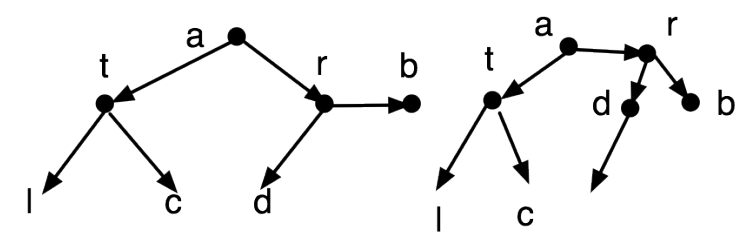

These differ only in the level of $r$. So we compute the new level accordingly, using the nlvl helper function.

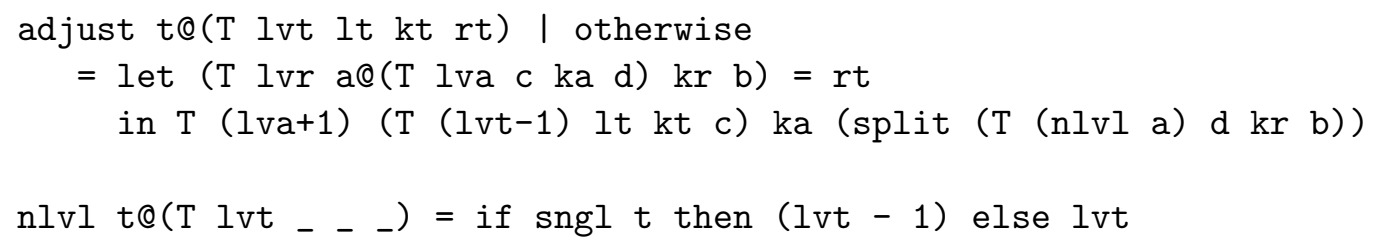

Here is the complete code for adjust, which is just the proper assemblage of the code fragments presented above.

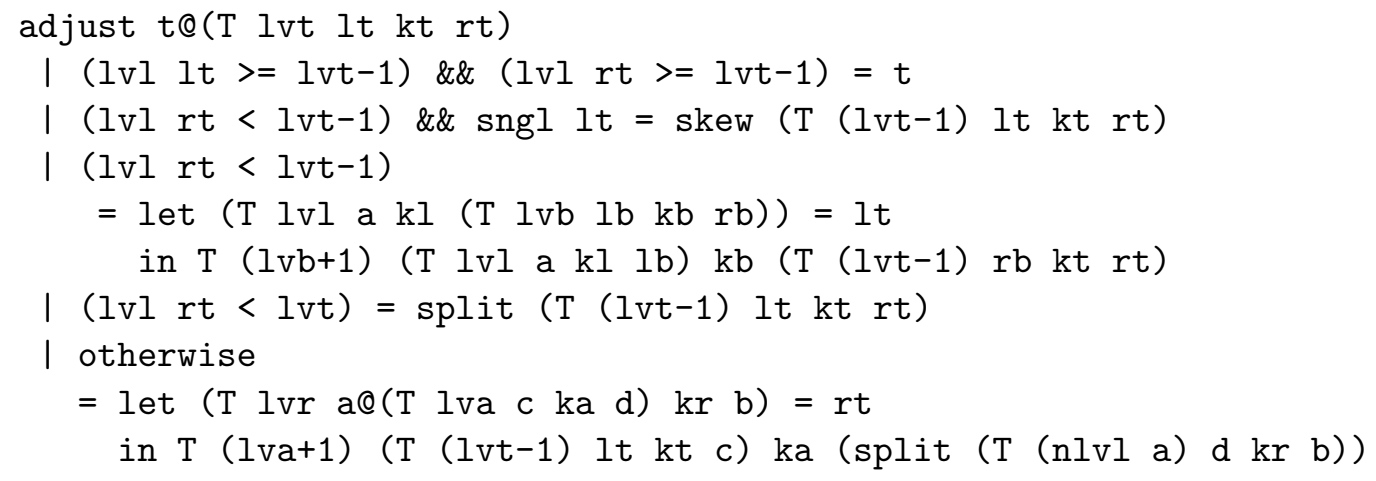

\section{Conclusions}

I use this presentation in a first "advanced level" course on computer science for students with good mathematical skills (not necessarily with prior computing experience). It is preceded by an early treatment of Braun trees [3] (which implement sequences and are the simplest useful data structure demonstrating logarithmic-depth trees) and of naive elementary binary search trees. When we return to the subject late in the term, we first discuss 2-3 and 2-3-4 trees, and insertion into red-black trees based on Okasaki's version. Deletion from AA trees is the last topic in the course.

Despite the fact that deletion from AA trees is simpler to explain and implement than deletion from any other flavour of balanced binary search trees, I would not recommend that it be covered in a first course taken by all computer science majors, or by non-majors. Since my course has non-majors enrolled, and I treat the topic so late, there is no assignment based on the material, and students are not responsible for it on the final exam. Consequently, it serves mainly to close the gap and fulfil the promise of an efficient implementation of maps, and as an advertisement for the more complicated invariants of data structures to be seen in later CS courses.

However, this topic fits well into a second-course treatment of balanced binary search trees, whether for majors or non-majors. Even in a course using a conventional imperative garbage-collected language 
for code examples, an initial immutable, recursive, functional treatment will improve students' understanding of the topic prior to covering the sort of code found in most textbooks and in library implementations.

\section{Bibliography}

\section{References}

[1] A. Aho, J. Hopcroft \& J. Ullman (1974): The Design And Analysis of Computer Programs. Addison-Wesley.

[2] A. Andersson (1993): Balanced search trees made simple. In: Proceedings of the 3rd Workshop on Algorithms and Data Structures, Springer Verlag, pp. 60-71, doi:10.1007/3-540-57155-8_236.

[3] W. Braun \& M. Rem (1983): A logarithmic implementation of flexible arrays. Technical Report MR83/4, Eindhoven Institute of Technology.

[4] (2014): Data.Set.RBTree. Available at http://hackage.haskell.org/package/llrbtree-0.1.1/ docs/src/Data-Set-RBTree.html.

[5] L. J. Guibas \& R. Sedgewick (1978): A dichromatic framework for balanced trees. In: Proceedings of the 19th Annual Symposium on Foundations of Computer Science, IEEE, pp. 8-21, doi:10.1109/SFCS.1978.3.

[6] (2014): Haskell. Available at http://www.haskell.org.

[7] S. Kahrs (2001): Red-black trees with types. Journal of Functional Programming 11(4), doi $10.1017 / \mathrm{S} 0956796801004026$

[8] S. Kahrs (2014): Untyped red-black trees. Available at http://www.cs.kent.ac.uk/people/staff/ smk/redblack/Untyped.hs

[9] M. Might (2014): Deletion from red-black trees: the missing method. Available at http://matt.might. net/articles/red-black-delete/.

[10] C. Okasaki (1999): Red-black trees in a functional setting. Journal of Functional Programming 9(4), doi $10.1017 /$ S0956796899003494

[11] (2014): Racket. Available at http://www.racket-lang.org 